\title{
The Static Maxwell System in Three
}

Dimensional Axially Symmetric Inhomogeneous Media and Axially Symmetric Generalization of the CauchyRiemann System

\section{Dmitry Bryukhov \& Uwe Kähler}

Advances in Applied Clifford Algebras

ISSN 0188-7009

Volume 27

Number 2

Adv. Appl. Clifford Algebras (2017) 27:993-1005

DOI 10.1007/s00006-016-0739-x

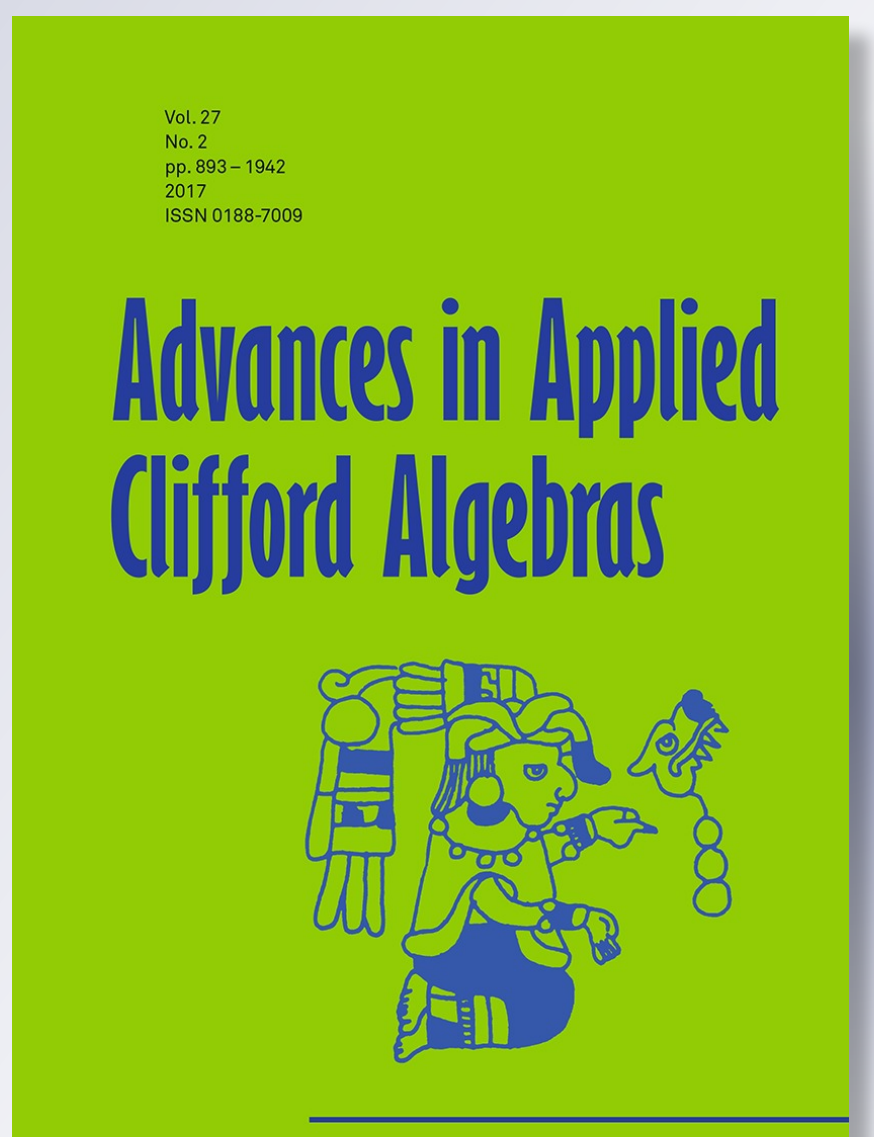

Birkhäuser 
Your article is protected by copyright and all rights are held exclusively by Springer International Publishing. This e-offprint is for personal use only and shall not be selfarchived in electronic repositories. If you wish to self-archive your article, please use the accepted manuscript version for posting on your own website. You may further deposit the accepted manuscript version in any repository, provided it is only made publicly available 12 months after official publication or later and provided acknowledgement is given to the original source of publication and a link is inserted to the published article on Springer's website. The link must be accompanied by the following text: "The final publication is available at link.springer.com". 


\title{
The Static Maxwell System in Three Dimensional Axially Symmetric Inhomogeneous Media and Axially Symmetric Generalization of the Cauchy-Riemann System
}

\author{
Dmitry Bryukhov and Uwe Kähler@* \\ To Vladislav Kravchenko, Heinz Leutwiler and Daniele Struppa \\ Communicated by Eckhard Hitzer
}

\begin{abstract}
In this paper we discuss different generalizations of the Cauchy-Riemann system and their connection with the static Maxwell system. In particular, this allows us to present relations between slicemonogenic functions and hypermonogenic functions, as well as to provide a physical interpretation of slice-monogenic functions. Furthermore, we present an explicit and complete set of basic solutions of a new class of axial-hypermonogenic functions in $\mathbb{R}^{3}$. In the end we determine the symmetry operators for the class of axial-hypermonogenic functions.
\end{abstract}

Mathematics Subject Classification. Primary 30G35; Secondary 78M25. Keywords. Inhomogeneous media, Reduced quaternionic variable, Slicemonogenic functions, Bessel equation.

\section{Introduction, Preliminaries, and Notations}

\subsection{Introduction and Preliminaries}

In 2010 Khmelnytskaya et al. [20] studied an important class of meridional electrostatic fields as analytic solutions of the static Maxwell system in axially symmetric inhomogeneous media in $\mathbb{R}^{3}=\left\{\left(x_{0}, x_{1}, x_{2}\right), x_{j} \in \mathbb{R}, j=0,1,2\right\}$ with dielectric permittivity $\varepsilon=\varepsilon\left(x_{0}, \sqrt{x_{1}^{2}+x_{2}^{2}}\right)$

$$
\left\{\begin{array}{l}
\operatorname{div}(\varepsilon \vec{E})=0 \\
\operatorname{curl} \vec{E}=0,
\end{array}\right.
$$

${ }^{*}$ Corresponding author. 
depending on the cylindrical radial variable $\sqrt{x_{1}^{2}+x_{2}^{2}}$ and the independent variable $x_{0}$. Hereby, div and curl denote the usual divergence and curl operators. There are several important special cases of system (1.1). First and foremost one has the well-known Riesz system or static Maxwell system in homogeneous media in $\mathbb{R}^{3}$ associated to a dielectric permittivity $\varepsilon=1$, whose solutions are the object of study in modern quaternionic analysis (see, e.g., $[18,28])$. This particular Riesz system written in terms of its components becomes (see, e.g., $[18,28])$ :

$$
\left\{\begin{array}{l}
\frac{\partial u_{0}}{\partial x_{0}}-\frac{\partial u_{1}}{\partial x_{\partial}}-\frac{\partial u_{2}}{\partial x_{2}}=0 \\
\frac{\partial u_{0}}{\partial x_{1}}=-\frac{\partial u_{1}}{\partial x_{0}}, \quad \frac{\partial u_{0}}{\partial x_{2}}=-\frac{\partial u_{2}}{\partial x_{0}}, \\
\frac{\partial u_{1}}{\partial x_{2}}=\frac{\partial u_{2}}{\partial x_{1}},
\end{array}\right.
$$

where $\vec{E}=\left(E_{0}, E_{1}, E_{2}\right):=\left(u_{0},-u_{1},-u_{2}\right)$.

We say that a scalar $C^{2}$-function $h=h\left(x_{0}, x_{1}, x_{2}\right)$ is a harmonic potential function associated to the $C^{1}$-vector field $\vec{E}$ if $u_{0}=\frac{\partial h}{\partial x_{0}}$ and $u_{j}=$ $-\frac{\partial h}{\partial x_{j}}, j=1,2$. More important, these first order systems arise from factorizing the Laplace operator by the Cauchy-Riemann and its adjoint operator.

In 1992 a modified quaternionic analysis was developed on the basis of the hyperbolic version of the Laplace equation in $\mathbb{R}^{3}[26,27]$

$$
x_{2} \Delta h-\frac{\partial h}{\partial x_{2}}=0 .
$$

In analogy to the previous systems its $C^{2}$-solutions $h$ were called hyperbolically harmonic functions. If $x_{2} \neq 0$ then equation (1.3) further reduces to

$$
x_{2} \Delta h-\frac{\partial h}{\partial x_{2}}=x_{2}^{2} \operatorname{div}\left(x_{2}^{-1} \operatorname{grad} h\right)=0 .
$$

The corresponding first-order system (see, e.g., [26-28]) has the form

$$
\left\{\begin{array}{l}
x_{2}\left(\frac{\partial u_{0}}{\partial x_{0}}-\frac{\partial u_{1}}{\partial x_{1}}-\frac{\partial u_{2}}{\partial x_{2}}\right)+u_{2}=0 \\
\frac{\partial u_{0}}{\partial x_{1}}=-\frac{\partial u_{1}}{\partial x_{0}}, \quad \frac{\partial u_{0}}{\partial x_{2}}=-\frac{\partial u_{2}}{\partial x_{0}}, \\
\frac{\partial u_{1}}{\partial x_{2}}=\frac{\partial u_{2}}{\partial x_{1}},
\end{array}\right.
$$

where again $\operatorname{grad} h=\vec{E}:=\left(u_{0},-u_{1},-u_{2}\right)$ for $C^{1}-$ components $u_{j}$. More important, under a simple change of dependent variables this system is a particular case of the static Maxwell system (1.1) in an inhomogeneous media with dielectric permittivity $\varepsilon=x_{2}{ }^{-1}[28]$.

Hereby, Leutwiler gave a new interpretation of the Fueter's construction for a class of functions of the reduced quaternionic variable associated with classical holomorphic functions under the special condition $x_{1} \frac{\partial h}{\partial x_{2}}=x_{2} \frac{\partial h}{\partial x_{1}}$ (see, e.g., $[4,5,26,27]$ ). Additionally, he constructed analytic solutions of (1.5) using properties of analytic functions of reduced quaternionic variables. In the early 2000 a class of reduced quaternion-valued hypermonogenic functions corresponding to the class of $C^{1}$-solutions of $(1.5)$ was described in $[11,12]$.

In this paper we investigate a similar type of Riesz system (or static Maxwell system in homogeneous media) associated to a dielectric permittivity 
depending on a cylindrical variable. Indeed, the first author (see, e.g., [46]) studied a new axially symmetric generalization of the Cauchy-Riemann system in $\mathbb{R}^{n+1}$, with particular emphasis to the case of $\mathbb{R}^{3}$. This new axially symmetric system takes the form:

$$
\left\{\begin{array}{l}
\left(x_{1}^{2}+x_{2}^{2}\right)\left(\frac{\partial u_{0}}{\partial x_{0}}-\frac{\partial u_{1}}{\partial x_{1}}-\frac{\partial u_{2}}{\partial x_{2}}\right)+\left(x_{1} u_{1}+x_{2} u_{2}\right)=0 \\
\frac{\partial u_{0}}{\partial x_{1}}=-\frac{\partial u_{1}}{\partial x_{0}}, \quad \frac{\partial u_{0}}{\partial x_{2}}=-\frac{\partial u_{2}}{\partial x_{0}}, \\
\frac{\partial u_{1}}{\partial x_{2}}=\frac{\partial u_{2}}{\partial x_{1}},
\end{array}\right.
$$

and it corresponds to the static Maxwell system in axially symmetric inhomogeneous media with dielectric permittivity depending on a cylindrical coordinate, that is, $\epsilon=\rho^{-1}:=\left(x_{1}^{2}+x_{2}^{2}\right)^{-1 / 2}$.

Assuming $\rho^{2}=x_{1}^{2}+x_{2}^{2} \neq 0$, this system is associated to the axially symmetric Laplace-Beltrami equation (see, e.g., [6]):

$$
\Delta_{B} h:=\left(x_{1}^{2}+x_{2}^{2}\right) \Delta h-\left(x_{1} \frac{\partial h}{\partial x_{1}}+x_{2} \frac{\partial h}{\partial x_{2}}\right)=0,
$$

where again $h$ as a potential is linked to the components $u_{s}, s=0,1,2$ via $\operatorname{grad} h=\vec{E}:=\left(u_{0},-u_{1},-u_{2}\right)$ for some $C^{1}$-components $u_{j}$. For $\rho=$ $\sqrt{x_{1}^{2}+x_{2}^{2}} \neq 0$ the axially symmetric Laplace-Beltrami equation (1.7) can be rewritten as

$$
\left(x_{1}^{2}+x_{2}^{2}\right) \Delta h-\left(x_{1} \frac{\partial h}{\partial x_{1}}+x_{2} \frac{\partial h}{\partial x_{2}}\right)=\rho^{3} \operatorname{div}\left(\rho^{-1} \operatorname{grad} h\right)=0 .
$$

The axially symmetric system (1.6) allow us to construct, in particular, analytic solutions of the static Maxwell system in $\mathbb{R}^{3}$ in axially symmetric inhomogeneous media with dielectric permittivity $\varepsilon=\rho^{-1}$.

One must add that both systems, (1.6) and (1.5), represent non-Euclidean modifications of $(1.2)[27,28]$. But in contrast to classical hyperbolic metric $d s^{2}=\frac{d x_{0}{ }^{2}+d x_{1}{ }^{2}+d x_{2}{ }^{2}}{x_{2}{ }^{2}}$ on the half-space $\left(x_{2}>0\right)$ which is closely connected with system (1.5) (see, e.g., [27,28]), system (1.6) is now associated to a new axially symmetric metric (see [6]) given, outside the axis $x_{0}$, by:

$$
d s^{2}=\frac{d x_{0}^{2}+d x_{1}^{2}+d x_{2}^{2}}{\rho^{2}}=\frac{d x_{0}^{2}+d \rho^{2}}{\rho^{2}}=\frac{d r^{2}}{r^{2}-x_{0}^{2}}=\frac{d r^{2}}{r^{2} \sin ^{2} \varphi} .
$$

In this paper we will show the connections of system (1.6) with other classical systems of quaternionic analysis, like slice-monogenic functions. This will lead to some remarks about the practical applicability of such systems. Furthermore, using the inherent symmetries of such we are going to construct basic solutions which allow us to represent all solutions of (1.6).

\subsection{Notations}

The real algebra of quaternions $\mathbb{H}$ is a four dimensional skew algebra over the real field generated by real unity 1 and three imaginary unities $i, j$, and $k$ satisfy to the following multiplication rules

$$
i^{2}=j^{2}=k^{2}=i j k=-1, \quad i j=-j i=k .
$$


An arbitrary quaternion is written as $q=x_{0}+i x_{1}+j x_{2}+k x_{3}$ and we define its scalar and vectorial parts as

$$
S c(q)=x_{0}, \quad \operatorname{Vec}(q)=i x_{1}+j x_{2}+k x_{3},
$$

respectively. Moreover, the conjugation of a quaternion is defined as the automorphism

$$
q \mapsto \bar{q}:=S c(q)-\operatorname{Vec}(q) .
$$

In such way, we obtain the Euclidean norm in $\mathbb{R}^{4}$

$$
\|q\|^{2}:=q \bar{q}=x_{0}^{2}+x_{1}^{2}+x_{2}^{2}+x_{3}^{2},
$$

and the following identification

$$
q=x_{0}+i x_{1}+j x_{2}+k x_{3} \sim\left(x_{0}, x_{1}, x_{2}, x_{3}\right)
$$

between $\mathbb{H}$ and $\mathbb{R}^{4}$ is valid. Also, for every non-zero quaternion $q$ a unique inverse exists, $q^{-1}=\bar{q} /\|q\|^{2}$.

We obtain the space of reduced quaternions by imposing $x_{3}=0$. Thus, the reduced quaternionic variable is represented by $x=x_{0}+i x_{1}+j x_{2}$ and, from now on, we identify it with the vector $\left(x_{0}, x_{1}, x_{2}\right) \in \mathbb{R}^{3}$.

We consider $\Omega \subset \mathbb{R}^{3}$ an open domain (with respect to the Euclidian metric). A reduced quaternionic function of reduced quaternionic variable will be represented as

$$
u\left(x_{0}, x_{1}, x_{2}\right)=u_{0}\left(x_{0}, x_{1}, x_{2}\right)+i u_{1}\left(x_{0}, x_{1}, x_{2}\right)+j u_{2}\left(x_{0}, x_{1}, x_{2}\right),
$$

where $u_{s}: \Omega \subset \mathbb{R}^{3} \rightarrow \mathbb{R}, s=0,1,2$. Moreover, properties such as continuity will be ascribed to $u$ if and only if all its components $u_{s}$ verify it, that is, $u \in C^{2}(\Omega)$ iff $u_{s} \in C^{2}(\Omega)$, for $s=0,1,2$.

We are now in conditions to characterize solutions of system (1.6):

Definition 1.1. Let $\Omega \subset \mathbb{R}^{3}$ be an open set. If $u=u_{0}+i u_{1}+j u_{2}: \Omega \rightarrow \mathbb{R}^{3}$ is in $C^{1}(\Omega)$ and its components satisfy (1.6) then we say that $u$ is an axialhypermonogenic function in $\Omega$.

We finalize the section with the notation used for our cylindrical coordinates. As seen,

$$
x=x_{0}+i x_{1}+j x_{2}=x_{0}+i \rho \cos \theta+j \rho \sin \theta,
$$

that is to say,

$$
x_{0}=x_{0}, \quad x_{1}=\rho \cos \theta, \quad x_{2}=\rho \sin \theta,
$$

with $\rho=\sqrt{x_{1}^{2}+x_{2}^{2}}$, and $\theta=\arctan \frac{x_{2}}{x_{1}}\left(x_{1} \neq 0\right)$. Moreover, we denote by

$$
r=\sqrt{x_{0}^{2}+x_{1}^{2}+x_{2}^{2}}=\sqrt{x_{0}^{2}+\rho^{2}}
$$

the norm of our reduced quaternionic variable $x$. 


\section{Two Generalizations of the Cauchy-Riemann System in $\mathbb{R}^{3}$ in Cylindrical Coordinates and its Connection with the Class of Slice-Monogenic Functions}

The differences and similarities of the above mentioned systems can best be seen in terms of cylindrical coordinates. This is due to the fact that it is closely connected with Fueter's construction [13] and the symmetry of system (1.6). Indeed, restricting to the meridional plane spanned by $\left(x_{0}, \rho\right)$ the Riesz system (1.2) becomes a Vekua-type system (see, e.g., [21]):

$$
\left\{\begin{array}{l}
\rho\left(\frac{\partial u_{0}}{\partial x_{0}}-\frac{\partial u_{\rho}}{\partial \rho}\right)-u_{\rho}=0 \\
\frac{\partial u_{0}}{\partial \rho}=-\frac{\partial u_{\rho}}{\partial x_{0}}
\end{array}\right.
$$

where

$$
u_{0}=\frac{\partial h}{\partial x_{0}}, \quad u_{\rho}=-\frac{\partial h}{\partial \rho} .
$$

In terms of Fueter's construction we have:

$$
F(x)=u_{0}(x)+i u_{1}(x)+j u_{2}(x)=u_{0}\left(x_{0}, \rho\right)+I u_{\rho}\left(x_{0}, \rho\right),
$$

with

$$
\begin{gathered}
u_{1}=\frac{x_{1}}{\rho} u_{\rho}\left(x_{0}, \rho\right)=u_{\rho}\left(x_{0}, \rho\right) \cos \theta, \\
u_{2}=\frac{x_{2}}{\rho} u_{\rho}\left(x_{0}, \rho\right)=u_{\rho}\left(x_{0}, \rho\right) \sin \theta .
\end{gathered}
$$

Furthermore, we also recall that the Laplace equation under conditions $\frac{\partial h}{\partial \theta}=$ $\frac{\partial^{2} h}{\partial \theta^{2}}=0$ can be represented in the form of the Weinstein equation

$$
\rho\left(\frac{\partial^{2} h}{\partial x_{0}{ }^{2}}+\frac{\partial^{2} h}{\partial \rho^{2}}\right)+\kappa \frac{\partial h}{\partial \rho}=0
$$

in the framework of generalized axially symmetric potential theory (GASPT, see e.g., $[10,16,19,31])$ with integer parameter $\kappa$.

Our system (1.6) can be considered as a Cauchy-Riemann system in the meridional plane spanned by $\left(x_{0}, \rho\right)$ :

$$
\left\{\begin{array}{l}
\frac{\partial u_{0}}{\partial x_{0}}-\frac{\partial u_{\rho}}{\partial \rho}=0, \\
\frac{\partial u_{0}}{\partial \rho}=-\frac{\partial u_{\rho}}{\partial x_{0}}
\end{array}\right.
$$

where, in terms of Fueter's construction, we have

$$
\begin{aligned}
& F(x)=u_{0}+i u_{1}+j u_{2}=u_{0}\left(x_{0}, \rho\right)+I u_{\rho}\left(x_{0}, \rho\right), \\
& u_{1}=u_{\rho}\left(x_{0}, \rho\right) \cos \theta, \quad u_{2}=u_{\rho}\left(x_{0}, \rho\right) \sin \theta, \quad u_{\rho}=-\frac{\partial h}{\partial \rho} .
\end{aligned}
$$

Hereby, one can easily see the difference with system (1.5) which does not have a nice form when restricted to the variables $\left(x_{0}, \rho\right)$. For convenience to the reader we just provided the following hyperbolic version of the Laplace equation (1.3):

$$
\sin ^{2} \theta\left(\frac{\partial^{2} h}{\partial x_{0}^{2}}+\frac{\partial^{2} h}{\partial \rho^{2}}\right)=0 .
$$


Let us give some remarks on the above systems, in particular about its connection with slice-monogenic functions in $\mathbb{R}^{3}$. In 2007 Gentili and Struppa $[9,14]$ defined Cullen regular functions (nowadays mainly called slicemonogenic or slice-regular functions, see, e.g. $[7,15])$. These are defined as reduced quaternion-valued functions $F$ which fulfill the following equation $D F=\left(\frac{\partial}{\partial x_{0}}+I \frac{\partial}{\partial \rho}\right) F=0$ on each slice domain belonging to the plane spanned by 1 and $I \in S^{2}$. Let us point out that a priori $D$ and $F$ are non-commutative which allows in fact to define left- and right-slice-monogenic functions [8].

Now, if we additionally impose $F$ being of the form $F=u_{0}\left(x_{0}, \rho\right)+$ $I u_{\rho}\left(x_{0}, \rho\right)$ then the above definition can be written as the Cauchy-Riemann system (2.5) in the meridional plane:

$$
\left\{\begin{array}{l}
\frac{\partial u_{0}}{\partial x_{0}}-\frac{\partial u_{\rho}}{\partial \rho}=0 \\
\frac{\partial u_{0}}{\partial \rho}=-\frac{\partial u_{\rho}}{\partial x_{0}}
\end{array}\right.
$$

which corresponds to Fueter's construction [13]. In 2008 Gürlebeck, Habetha and Sprößig [17] described basic analytical properties of elementary radially holomorphic functions in $\mathbb{R}^{n+1}$, in particular the paravector-valued powers and the exponential function. Here radially holomorphic functions also correspond to Fueter's construction and is also another way of looking at slicemonogenic functions of the special type (2.5).

For us it is important to point out that the class of slice-monogenic functions can be physically interpreted as the class of solutions of the static Maxwell system in axially symmetric inhomogeneous medium with dielectric permittivity $\varepsilon=\rho^{-1}$ in $\mathbb{R}^{3}$, thus, characterizing meridional electrostatic fields in terms of [20].

Let us look at some examples generated by specific potentials which are standard examples in the modeling of electrostatic potentials.

Example 1. The function $\overline{f(x)}=\overline{x^{-1}}=\operatorname{grad} h, x \neq 0$, conjugated to the reduced quaternionic inversion describes the classical inversion transformation in $\mathbb{R}^{3}$. The inversion transformation can be interpreted as an electrostatic field of a single isolated positive electric charge in the case of the static Maxwell system in inhomogeneous medium with dielectric permittivity $\varepsilon=\rho^{-1}$ in $\mathbb{R}^{3}$. The potential function has the following form: $h\left(x_{0}, x_{1}, x_{2}\right)=\ln |x|+C=\frac{1}{2} \ln \left(x_{0}^{2}+x_{1}^{2}+x_{2}^{2}\right)+C \quad(\mathrm{C}$ is an arbitrary constant).

Example 2. Consider the $n$-th power as function of the reduced quaternionic variable $F(x)=x^{n}$. The restriction to the unit sphere $S^{2}$ in $\mathbb{R}^{3}$ can be characterized as the reduced quaternionic form of the exponential function $e^{I n \varphi}$ on $S^{2}$, instead of the unit circle $S^{1}$ in classical complex analysis (see, e.g., [18] in modern quaternionic analysis).

Functions $x^{n}$ and $\overline{x^{n}}$ map the unit sphere $S^{2}$ to itself in $\mathbb{R}^{3}$.

Corresponding meridional electrostatic fields:

$\overline{F(x)}=\overline{x^{n}}=r^{n}(\cos n \varphi-i \sin n \varphi \cos \theta-j \sin n \varphi \sin \theta)$.

The potential function has the following form:

$h\left(x_{0}, x_{1}, x_{2}\right)=r^{n+1} \cos (n+1) \varphi+C$ (C is an arbitrary constant $)$. 
On the other side, $x^{-n}=r^{-n}[\cos (-n \varphi)+i \sin (-n \varphi) \cos \theta+j \sin (-n \varphi) \sin \theta]$. Corresponding meridional electrostatic fields:

$\overline{x^{-n}}=r^{-n}(\cos n \varphi+i \sin n \varphi \cos \theta+j \sin n \varphi \sin \theta)$.

Functions $x^{n}$ and $\overline{x^{n}}$ map the interior of the unit ball $B^{3}$ to itself in $\mathbb{R}^{3}$, whereas functions $x^{-n}$ and $\overline{x^{-n}}$ map the interior of the unit ball $B^{3}$ to the exterior of $B^{3}$.

The potential function for electrostatic fields $\overline{x^{-n}}, n \neq 1$, has the following form: $h\left(x_{0}, x_{1}, x_{2}\right)=r^{-n+1} \cos (-n+1) \varphi+C$ ( $\mathrm{C}$ is an arbitrary constant).

Example 3. The exponential function of the reduced quaternionic variable $F(x)=e^{\gamma x}=e^{\gamma x_{0}}(\cos (\gamma \rho)+I \sin (\gamma \rho))$, where $\gamma \in \mathbb{R}$.

Corresponding meridional electrostatic fields:

$\overline{F(x)}=\overline{e^{\gamma x}}=e^{\gamma x_{0}}(\cos (\gamma \rho)-I \sin (\gamma \rho))$.

Functions $e^{\gamma x}$ and $\overline{e^{\gamma x}}$ map the horizontal plane $x_{0}=0$ to the sphere of radius $e^{\gamma x_{0}}$ in $\mathbb{R}^{3}$. Respectively functions $e^{\gamma x}$ and $\overline{e^{\gamma x}}$ map the plane $x_{0}=a=$ const to the sphere of radius $e^{\gamma a}$ in $\mathbb{R}^{3}$.

The potential function has the following form:

$h\left(x_{0}, x_{1}, x_{2}\right)=e^{\gamma x_{0}} \cos (\gamma \rho)+C$ ( $\mathrm{C}$ is an arbitrary constant $)$.

\section{Explicit Representations of a General Class of Solutions of the Static Maxwell System in Inhomogeneous Medium with Dielectric Permittivity $\varepsilon=\rho^{-1}$}

As was mentioned in the beginning in 2010 Khmelnytskaya et al. [20] studied properties of class of the meridional electrostatic fields, and additionally studied properties of class of the transverse electrostatic fields as analytic solutions of the static Maxwell system in axially symmetric inhomogeneous media.

In the case of meridional fields the vector $\vec{E}$ is independent of the angular coordinate $\theta$, that is, the component $E_{\theta}$ of the vector $\vec{E}$ vanishes identically. This means that the vector of such field belongs to a plane spanned by the axis $x_{0}$ and the distance described by the cylindrical radial variable. The field then is completely described by a two-component vector-function in the meridional plane.

In the other case we have the condition that the vector $\vec{E}$ is independent of $x_{0}$ and the first component vanishes. The vector representing such a field belongs to a plane perpendicular to the axis $x_{0}$ and the corresponding model is described by a $2 \mathrm{D}$-vector in the plane $\left(x_{1}, x_{2}\right)$.

Thus, the above statement describes the physical meaning of the cylindrical radial variable in both cases of system (1.5) and system (1.6). Furthermore, under the special condition $x_{1} \frac{\partial h}{\partial x_{2}}=x_{2} \frac{\partial h}{\partial x_{1}}$ and $x_{2} \neq 0$ solutions of the system (1.5) are solutions of the system (1.6) and vice versa.

Our examples of elementary functions of the reduced quaternionic variable, associated with classical holomorphic functions, represent meridional fields $\vec{E}=\left(E_{0}, E_{1}, E_{2}\right)$ of the static Maxwell system (1.1) in inhomogeneous medium with dielectric permittivity $\varepsilon=\rho^{-1}$ : In particular, we have to deal 
with meridional fields of positive and negative single charges. Potential functions $h=h\left(x_{0}, x_{1}, x_{2}\right)$ have the physical meaning of electrostatic potentials.

More general models of electrostatic fields in inhomogeneous media in the form of analytical solutions of the static Maxwell system are very interesting in many applications (see, e.g., [23,24]). In particular, problems of refraction in inhomogeneous dielectric media are actual in geometrical optics (see, e.g., [3]). This connection with slice-monogenic functions allows to adapt the ideas of [25] to the present case which we are planning to do in future work.

To construct basic solutions of system (1.6) we proceed in the usual way by separation of variables $\left\{\left(x_{0}, \rho\right)\right\}$ and $\{(\theta)\}$ (see, e.g., $[1,2,29]$ ).

Since the Laplace operator in $\mathbb{R}^{3}$ in cylindrical coordinates for $h\left(x_{0}, \rho, \theta\right)$ $=g\left(x_{0}, \rho\right) s(\theta)$ is given by

$$
\Delta h=s(\theta) \frac{\partial^{2} g}{\partial x_{0}^{2}}+s(\theta) \frac{\partial^{2} g}{\partial \rho^{2}}+\frac{s(\theta)}{\rho} \frac{\partial g}{\partial \rho}+\frac{g}{\rho^{2}} \frac{\partial^{2} s}{\partial \theta^{2}},
$$

we obtain for the axially symmetric Laplace-Beltrami equation (1.7) in $\mathbb{R}^{3}$ in cylindrical coordinates under condition $h\left(x_{0}, \rho, \theta\right)=g\left(x_{0}, \rho\right) s(\theta)$ :

$$
s(\theta) \rho^{2}\left(\frac{\partial^{2} g}{\partial x_{0}^{2}}+\frac{\partial^{2} g}{\partial \rho^{2}}\right)+g \frac{\partial^{2} s}{\partial \theta^{2}}=0 .
$$

If $g\left(x_{0}, \rho\right) s(\theta) \neq 0$ we get

$$
\frac{\rho^{2}}{g}\left(\frac{\partial^{2} g}{\partial x_{0}^{2}}+\frac{\partial^{2} g}{\partial \rho^{2}}\right)=-\frac{1}{s(\theta)} \frac{\partial^{2} s}{\partial \theta^{2}}=\lambda^{2} \quad(\lambda=\text { const } \in \mathbb{R}) .
$$

Then Eq. (3.2) is equivalent to the following system of equations

$$
\left\{\begin{array}{l}
\frac{\partial^{2} g}{\partial x_{0}^{2}}+\frac{\partial^{2} g}{\partial \rho^{2}}-\frac{\lambda^{2}}{\rho^{2}} g=0 \\
\frac{d^{2} s(\theta)}{d \theta^{2}}+\lambda^{2} s(\theta)=0
\end{array}\right.
$$

In particular, solutions of the second equation in (3.3) have the wellknown form $s(\theta)=C_{1} \cos \lambda \theta+C_{2} \sin \lambda \theta$. Additionally, the periodicity of $s$ implies $\lambda \in \mathbb{Z}$.

For the first equation in (3.3) let us consider solutions in the form: $g\left(x_{0}, \rho\right)=e^{\beta x_{0}} \Upsilon(\rho), \beta=$ const $\in \mathbb{R}$, then

$$
\frac{\partial^{2} g}{\partial x_{0}{ }^{2}}+\frac{\partial^{2} g}{\partial \rho^{2}}-\frac{\lambda^{2}}{\rho^{2}} g=\beta^{2} e^{\beta x_{0}} \Upsilon(\rho)+e^{\beta x_{0}} \frac{\partial^{2} \Upsilon(\rho)}{\partial \rho^{2}}-\frac{\lambda^{2}}{\rho^{2}} e^{\beta x_{0}} \Upsilon(\rho)=0
$$

or, since $\Upsilon(\rho)$ is a function of one real variable $\rho$, in the equivalent form:

$$
\rho^{2} \frac{d^{2} \Upsilon(\rho)}{d \rho^{2}}+\left(\beta^{2} \rho^{2}-\lambda^{2}\right) \Upsilon(\rho)=0 .
$$

Changing $\Upsilon(\rho)$ into a function $\Upsilon_{1}\left(\rho_{1}\right)$ according to $\rho=\beta \rho_{1}{ }^{-1}, \Upsilon=$ $\beta^{-\frac{1}{2}} \rho_{1}^{\frac{1}{2}} \Upsilon_{1}$ (see, e.g., $[22,30]$ ) allows us to transform the linear differential equation (3.5) to a Bessel equation:

$$
\rho_{1}^{2} \frac{d^{2} \Upsilon_{1}\left(\rho_{1}\right)}{d \rho_{1}{ }^{2}}+\rho_{1} \frac{d \Upsilon_{1}\left(\rho_{1}\right)}{d \rho_{1}}+\left(\rho_{1}^{2}-\lambda^{2}-\frac{1}{4}\right) \Upsilon_{1}\left(\rho_{1}\right)=0
$$


Its linear independent solutions are $\Upsilon_{1}\left(\rho_{1}\right)=A_{1} J_{\frac{\sqrt{4 \lambda^{2}+1}}{2}}\left(\rho_{1}\right)+A_{2}$ $J_{-\frac{\sqrt{4 \lambda^{2}+1}}{2}}\left(\rho_{1}\right)$ which leads to the solution

$$
\Upsilon(\rho)=A_{1} \sqrt{\frac{2 \beta}{\pi}} \frac{1}{\rho} j_{\frac{\sqrt{4 \lambda^{2}+1}-1}{2}}\left(\frac{\beta}{\rho}\right)+A_{2}(-1)^{n+1} \sqrt{\frac{2 \beta}{\pi}} \frac{1}{\rho} y_{\frac{\sqrt{4 \lambda^{2}+1}-1}{2}}\left(\frac{\beta}{\rho}\right)
$$

with $j_{n}$ and $y_{n}$ denoting the spherical Bessel functions of the first kind.

This leads to the following theorem.

Theorem 3.1. Basic solutions of the axially symmetric Laplace-Beltrami equation (1.8) are given in cylindrical coordinates by

$$
h_{\beta}\left(x_{0}, \rho, \theta\right)=\sum_{\lambda=0}^{\infty} e^{\beta x_{0}} g_{\beta}(\rho) s_{\beta}(\theta)
$$

with

$$
g_{\beta}(\rho)=\sqrt{\frac{2 \beta}{\pi}} \frac{1}{\rho}\left(A_{1, \lambda} j_{\frac{\sqrt{4 \lambda^{2}+1}-1}{2}}\left(\frac{\beta}{\rho}\right)+A_{2, \lambda} y_{\frac{\sqrt{4 \lambda^{2}+1}-1}{2}}\left(\frac{\beta}{\rho}\right)\right)
$$

and

$$
s_{\beta}(\theta)=\left(C_{1, \lambda} \cos (\lambda \theta)+C_{2, \lambda} \sin (\lambda \theta)\right)
$$

for $\beta \in \mathbb{R}$ and $A_{1, \lambda}, A_{2, \lambda}, C_{1, \lambda}, C_{2, \lambda}$ being constants.

The above theorem allows us to present special solutions for system (1.6). Since we have

$$
\begin{aligned}
\partial_{\rho} g_{\beta}(\rho)= & \sqrt{\frac{2 \beta}{\pi}}\left(\left(-\frac{1}{\rho^{2}}\right)\left(A_{1, \lambda} j_{\frac{\sqrt{4 \lambda^{2}+1}-1}{2}}\left(\frac{\beta}{\rho}\right)+A_{2, \lambda} y_{\frac{\sqrt{4 \lambda^{2}+1}-1}{2}}\left(\frac{\beta}{\rho}\right)\right)\right. \\
& -\frac{\beta}{\rho^{3}}\left(A_{1, \lambda}\left(j_{\frac{\sqrt{4 \lambda^{2}+1}-3}{2}}\left(\frac{\beta}{\rho}\right)-\frac{(n+1) \rho}{\beta} j_{\frac{\sqrt{4 \lambda^{2}+1}-1}{2}}\left(\frac{\beta}{\rho}\right)\right)\right. \\
& \left.\left.-A_{2, \lambda}\left(y_{\frac{\sqrt{4 \lambda^{2}+}-3}{2}}\left(\frac{\beta}{\rho}\right)-\frac{(n+1) \rho}{\beta} y_{\frac{\sqrt{4 \lambda^{2}+1}-1}{2}}\left(\frac{\beta}{\rho}\right)\right)\right)\right)
\end{aligned}
$$

we get the following theorem.

Theorem 3.2. Basic solutions of system (1.6) have the form

$$
\begin{aligned}
u_{0}\left(x_{0}, \rho, \theta\right)= & \beta \sum_{\lambda=0}^{\infty} e^{\beta x_{0}} g_{\beta}(\rho) s_{\beta}(\theta) \\
u_{1}\left(x_{0}, \rho, \theta\right)= & \sum_{\lambda=0}^{\infty} e^{\beta x_{0}} \sqrt{\frac{2 \beta}{\pi}} \frac{1}{\rho^{2}}(\cos (\theta) \\
& \times\left(-\left(A_{1, \lambda} j \frac{\sqrt{4 \lambda^{2}+1}-1}{2}\left(\frac{\beta}{\rho}\right)+A_{2, \lambda} y \frac{\sqrt{4 \lambda^{2}+1}-1}{2}\left(\frac{\beta}{\rho}\right)\right)\right. \\
& -\frac{\beta}{\rho}\left(A_{1, \lambda}\left(j \frac{\sqrt{4 \lambda^{2}+1}-3}{2}\left(\frac{\beta}{\rho}\right)-\frac{(n+1) \rho}{\beta} j \frac{\sqrt{4 \lambda^{2}+1}-1}{2}\left(\frac{\beta}{\rho}\right)\right)\right. \\
& \left.\left.-A_{2, \lambda}\left(y \frac{\sqrt{4 \lambda^{2}+}-3}{2}\left(\frac{\beta}{\rho}\right)-\frac{(n+1) \rho}{\beta} y \frac{\sqrt{4 \lambda^{2}+1}-1}{2}\left(\frac{\beta}{\rho}\right)\right)\right)\right) \\
& \left.\times\left(C_{1, \lambda} \cos (\lambda \theta)+C_{2, \lambda} \sin (\lambda \theta)\right)\right)
\end{aligned}
$$




$$
\begin{aligned}
& -\sin \theta\left(\left(A_{1, \lambda} j \frac{\sqrt{4 \lambda^{2}+1}-1}{2}\left(\frac{\beta}{\rho}\right)+A_{2, \lambda} y \frac{\sqrt{4 \lambda^{2}+1}-1}{2}\left(\frac{\beta}{\rho}\right)\right)\right) \\
& \left.\times\left(-C_{1, \lambda} \sin (\lambda \theta)+C_{2, \lambda} \cos (\lambda \theta)\right)\right) \\
u_{2}\left(x_{0}, \rho, \theta\right)= & \sum_{\lambda=0}^{\infty} e^{\beta x_{0}} \sqrt{\frac{2 \beta}{\pi}} \frac{1}{\rho^{2}}(\sin (\theta) \\
& \times\left(-\left(A_{1, \lambda} \frac{\sqrt{4 \lambda^{2}+1}-1}{2}\left(\frac{\beta}{\rho}\right)+A_{2, \lambda} y \frac{\sqrt{4 \lambda^{2}+1}-1}{2}\left(\frac{\beta}{\rho}\right)\right)\right. \\
& -\frac{\beta}{\rho}\left(A_{1, \lambda}\left(j \frac{\sqrt{4 \lambda^{2}+1}-3}{2}\left(\frac{\beta}{\rho}\right)-\frac{(n+1) \rho}{\beta} j_{\frac{\sqrt{4 \lambda^{2}+1}-1}{2}}\left(\frac{\beta}{\rho}\right)\right)\right. \\
& \left.\left.-A_{2, \lambda}\left(y \frac{\sqrt{4 \lambda^{2}+}-3}{2}\left(\frac{\beta}{\rho}\right)-\frac{(n+1) \rho}{\beta} y \frac{\sqrt{4 \lambda^{2}+1}-1}{2}\left(\frac{\beta}{\rho}\right)\right)\right)\right) \\
& \left.\times\left(C_{1, \lambda} \cos (\lambda \theta)+C_{2, \lambda} \sin (\lambda \theta)\right)\right) \\
& +\cos \theta\left(\left(A_{1, \lambda} j \frac{\sqrt{4 \lambda^{2}+1}-1}{2}\left(\frac{\beta}{\rho}\right)+A_{2, \lambda} y \frac{\sqrt{4 \lambda^{2}+1}-1}{2}\left(\frac{\beta}{\rho}\right)\right)\right) \\
& \left.\left.\times\left(-C_{1, \lambda} \sin (\lambda \theta)+C_{2, \lambda} \cos (\lambda \theta)\right)\right)\right) .
\end{aligned}
$$

\section{First-Order Symmetries}

The construction of basic solutions in the previous chapter raises the question if these solutions generate all possible solutions. To answer this question we take a look at the first order symmetries, i.e. at all operators $L$ given by

$$
L u=A_{0}(x) \frac{\partial u}{\partial x_{0}}+A_{1}(x) \frac{\partial u}{\partial x_{1}}+A_{2}(x) \frac{\partial u}{\partial x_{2}}+B(x),
$$

such that

$$
\left[\Delta_{B}, L\right] u:=\left(\Delta_{B} L-L \Delta_{B}\right) u=M(x) \Delta_{B} u
$$

with an arbitrary first-order differential operator $M(x)$.

For the first term we get

$$
\begin{aligned}
{\left[\Delta_{B}, L\right] u=} & \left(2\left(x_{1}^{2}+x_{2}^{2}\right) \frac{\partial A_{1}}{\partial x_{1}}-2 x_{1} A_{1}-2 x_{2} A_{2}\right) \frac{\partial^{2} u}{\partial x_{1}^{2}} \\
& +\left(2\left(x_{1}^{2}+x_{2}^{2}\right) \frac{\partial A_{2}}{\partial x_{2}}-2 x_{1} A_{1}-2 x_{2} A_{2}\right) \frac{\partial^{2} u}{\partial x_{2}^{2}} \\
& +\left(2\left(x_{1}^{2}+x_{2}^{2}\right) \frac{\partial A_{0}}{\partial x_{0}}-2 x_{1} A_{1}-2 x_{2} A_{2}\right) \frac{\partial^{2} u}{\partial x_{2}^{2}} \\
& +2\left(\frac{\partial A_{1}}{\partial x_{2}}+\frac{\partial A_{2}}{\partial x_{1}}\right) \frac{\partial^{2} u}{\partial x_{1} \partial x_{2}}+2\left(\frac{\partial A_{0}}{\partial x_{1}}+\frac{\partial A_{1}}{\partial x_{0}}\right) \frac{\partial^{2} u}{\partial x_{0} \partial x_{1}} \\
& +\left(x_{1}^{2}+x_{2}^{2}\right)\left(2\left(\frac{\partial A_{0}}{\partial x_{2}}+\frac{\partial A_{2}}{\partial x_{0}}\right) \frac{\partial^{2} u}{\partial x_{0} \partial x_{2}}\right)
\end{aligned}
$$




$$
\begin{aligned}
& +\left(\left(x_{1}^{2}+x_{2}^{2}\right)\left(\Delta A_{1}+2 \frac{\partial B}{\partial x_{0}}\right)-x_{1} \frac{\partial A_{0}}{\partial x_{1}}-x_{2} \frac{\partial A_{0}}{\partial x_{2}}\right) \frac{\partial u}{\partial x_{0}} \\
& +\left(\left(x_{1}^{2}+x_{2}^{2}\right)\left(\Delta A_{1}+2 \frac{\partial B}{\partial x_{1}}\right)-x_{1} \frac{\partial A_{1}}{\partial x_{1}}-x_{2} \frac{\partial A_{1}}{\partial x_{2}}-A_{1}\right) \frac{\partial u}{\partial x_{1}} \\
& +\left(\left(x_{1}^{2}+x_{2}^{2}\right)\left(\Delta A_{1}+2 \frac{\partial B}{\partial x_{2}}\right)-x_{1} \frac{\partial A_{2}}{\partial x_{1}}-x_{2} \frac{\partial A_{2}}{\partial x_{2}}-A_{2}\right) \frac{\partial u}{\partial_{x_{2}}} \\
& +\left(x_{1}^{2}+x_{2}^{2}\right) \Delta B
\end{aligned}
$$

while for the second term we have $M(x)\left(\left(x_{1}^{2}+x_{2}^{2}\right)\left(\frac{\partial^{2} u}{\partial x_{0}^{2}}+\frac{\partial^{2} u}{\partial x_{1}^{2}} \frac{\partial^{2} u}{\partial x_{2}^{2}}\right)-x_{1} \frac{\partial u}{\partial x_{1}}-\right.$ $\left.x_{2} \frac{\partial u}{\partial x_{2}}\right)$.

Solving the system arising from the independence of the partial derivatives of $u$ we get as generators for the corresponding Lie algebra the operators $L_{1}=\frac{\partial}{\partial x_{0}}$ and $L_{2}=x_{1} \frac{\partial}{\partial x_{2}}-x_{2} \frac{\partial}{\partial x_{1}}=\frac{\partial}{\partial \theta}$.

In the same way for system (1.6) we get the symmetry operators $L_{1}=$ $\frac{\partial}{\partial x_{0}}$ and $L_{3}=x_{1} \frac{\partial}{\partial x_{2}}-x_{2} \frac{\partial}{\partial x_{1}}+i j=\frac{\partial}{\partial \theta}+i j$ as generators. Since these operators generated the whole algebra we get that all solutions of system (1.6) and solutions belonging to the kernel of the axially symmetric Laplace-Beltrami operator are generated by the basic solutions constructed in the previous section. Additionally, we would like to remark that in view of our observation of the correspondence between system (1.6) and slice-monogenic functions the above symmetry operators also represent the symmetry operators for slicemonogenic functions.

\section{Acknowledgements}

This work was supported by Portuguese funds through the CIDMA - Center for Research and Development in Mathematics and Applications, and the Portuguese Foundation for Science and Technology ("FCT-Fundação para a Ciência e a Tecnologia"), within project UID/MAT/0416/2013. The authors wish to thank the referees for valuable comments and suggestions.

\section{References}

[1] Aksenov, A.V.: Linear differential relations between solutions of the class of Euler-Poisson-Darboux equations. J. Math. Sci. 130(5), 4911-4940 (2005)

[2] Aramanovich, I.G., Levin, V.I.: Equations of Mathematical Physics [in Russian], 2nd edn. Nauka, Moscow (1969)

[3] Born, M., Wolf, E.: Principles of Optics, 7th edn. Cambridge University Press, Cambridge (2003)

[4] Bryukhov, D.A.: Axially symmetric generalization of the Cauchy-Riemann system and modified Clifford analysis (2003). arXiv:math/0302186v1 [math.CV]

[5] Bryukhov, D.: The independent and dependent reduced quaternionic variables in $\mathbb{R}^{3}$ and some applications. In: Proceedings of the ICCA9, Bauhaus University, Weimar (2011) 
[6] Bryukhov, D.: Modified Quaternionic Analysis in $\mathbb{R}^{3}$ and Generalizations of Conformal Mappings of the Second Kind. In: AIP Conference Proceedings, vol. 1389, New York, pp. 248-251 (2011)

[7] Colombo, F., Sabadini, I., Struppa, D.C.: Slice monogenic functions. Isr. J. Math. 171, 385-403 (2009)

[8] Colombo, F., Sabadini, I., Struppa, D.C.: Noncommutative Functional Calculus: Theory and Applications of Slice Hyperholomorphic Functions. Birkhäuser, Basel (2011)

[9] Cullen, C.G.: An integral theorem for analytic intrinsic functions on quaternions. Duke Math. J. 32, 139-148 (1965)

[10] Erdelyi, A.: Singularities of generalized axially symmetric potentials. Commun. Pure Appl. Math. 9, 403-414 (1956)

[11] Eriksson-Bique, S.-L., Leutwiler, H.: Hypermonogenic functions. In: Clifford Algebras and Their Applications in Mathematical Physics, vol. 2, pp. 287-302. Birkhäuser, Boston (2000)

[12] Eriksson-Bique, S.-L., Leutwiler, H.: Hypermonogenic functions and Möbius transformations. Adv. Appl. Clifford Algebras 11(S2), 67-76 (2001)

[13] Fueter, R.: Die Funktionentheorie der Differentialgleichungen $\Delta u=0$ und $\Delta \Delta u=0$ in vier reellen Veränderlichen. Comment. Math. Helv. 7(1), 307-330 (1934)

[14] Gentili, G., Struppa, D.C.: A new theory of regular functions of a quaternionic variable. Adv. Math. 216(1), 279-301 (2007)

[15] Ghiloni, R., Perotti, A.: Slice regular functions on real alternative algebras. Adv. Math. 226(2), 1662-1691 (2011)

[16] Gilbert, R.P.: On the singularities of generalized axially symmetric potentials. J. Ration. Mech. Anal. 6(1), 171-176 (1960)

[17] Gürlebeck, K., Habetha, K., Sprößig, W.: Holomorphic Functions in the Plane and n-Dimensional Space. Birkhäuser, Basel (2008)

[18] Gürlebeck, K., Morais, J.: On orthonormal polynomial solutions of the Riesz system in $\mathbb{R}^{3}$. In: Recent Advances in Computational and Applied Mathematics, pp. 143-158. Springer, Dordrecht (2011)

[19] Henrici, R.P.: On the domain of regularity of generalized axially symmetric potentials. Proc. Am. Math. Soc. 8(1), 29-31 (1957)

[20] Khmelnytskaya, K.V., Kravchenko, V.V., Oviedo, H.: On the solution of the static Maxwell system in axially symmetric inhomogeneous media. Math. Methods Appl. Sci. 33(4), 439-447 (2010)

[21] Kou, K.I., Qian, T., Sommen, F.: Generalizations of Fueter's theorem. Methods Appl. Anal. 9(2), 273-289 (2002)

[22] Krasnov, M.L., Kiselev, A.I., Makarenko, G.I.: Ordinary Differential Equations [in Russian], 4th edn. Editorial URSS, Moscow (2002)

[23] Kravchenko, V.V.: Applied Quaternionic Analysis, Research and Exposition in Mathematics Series, vol. 28. Heldermann Verlag, Lemgo (2003)

[24] Kravchenko, V.V.: Applied Pseudoanalytic Function Theory, Series: Frontiers in Mathematics. Birkhäuser, Basel (2009)

[25] Lavrentiev, M.A., Shabat, B.V.: Methods of the Theory of Functions of Complex Variable. Nauka, Moscow (1973) 
[26] Leutwiler, H.: Modified Clifford analysis. Complex Var. Theory Appl. 17, 153171 (1992)

[27] Leutwiler, H.: Modified quaternionic analysis in $\mathbb{R}^{3}$. Complex Var. Theory Appl. 20, 19-51 (1992)

[28] Leutwiler, H.: Quaternionic Analysis in $\mathbb{R}^{3}$ Versus its Hyperbolic Modification. NATO Science Series II: Mathematics. Physics and Chemistry, vol. 25, pp. 193-211. Kluwer, Dordrecht (2001)

[29] Miller Jr., W.: Symmetry and Separation of Variables. Addison-Wesley, Reading (1977)

[30] Watson, G.N.: A Treatise on the Theory of Bessel Functions, 2nd edn. Cambridge University Press, Cambridge (1944)

[31] Weinstein, A.: Generalized axially symmetric potential theory. Bull. Am. Math. Soc. 59(1), 20-38 (1953)

Dmitry Bryukhov

Magnit Ltd

Mira Avenue 17, Fryazino

Moscow Region

141196

Russia

e-mail: bryukhov@mail.ru

Uwe Kähler

Department of Mathematics

CIDMA-Center for Research and Development in Mathematics and Applications University of Aveiro

Aveiro

Portugal

e-mail: ukaehler@ua.pt

Received: March 31, 2016.

Accepted: November 9, 2016. 